\title{
Aggregation Modes of Anionic Oxacarbocyanines with Polycations in Solution and in ESAMs
}

\author{
Andrea Lodi and Glauco Ponterini \\ Dipartimento di Chimica, Università di Modena e Reggio Emilia and INSTM, Via Campi 183, Modena 41100, Italy
}

Received 30 June 2006; Revised 13 August 2006; Accepted 6 September 2006

\begin{abstract}
Interaction of oxacarbocyanines D-G with three polycations in aqueous solutions results in the formation of two types of likely small, distorted aggregates rather than the classical J aggregates. On the contrary, the latter are extensively and almost exclusively obtained in electrostatically self-assembled multilayers (ESAMs) prepared by alternate polycation/dye adsorption on quartz substrates. The J-aggregate growth on supported polycations is qualitatively shared by the four cyanines, a fact that reveals the crucial role of the double anionic substitutions on the dyes. On the other hand, films with $\mathrm{D}$ and $\mathrm{E}$, which are known to have a stronger tendency to give dimers in water, exhibit higher J-band intensities and stability upon drying relative to those with F and G. Based on these observations, we suggest that energetic factors associated with cofacial dye/dye van der Waals interactions, ultimately related with the degree of planarity of the conjugated chromophores, may still play a major role in controlling aggregation equilibria in these complex systems.
\end{abstract}

Copyright ( $) 2006$ A. Lodi and G. Ponterini. This is an open access article distributed under the Creative Commons Attribution License, which permits unrestricted use, distribution, and reproduction in any medium, provided the original work is properly cited.

\section{INTRODUCTION}

$\mathrm{H}$ and $\mathrm{J}$ aggregates of organic dyes have been known for 70 years $[1,2]$, but they have received a huge renewed attention in the last decades because of their unique spectroscopic (e.g., extremely narrow bandwidths) and photophysical properties (e.g., superradiance) which have stimulated both fundamental studies and the search for a myriad of possible technological applications, mostly as materials for optics and optoelectronics (see [3] and, for some recent reports, [4-9]).

We have recently shown that $\mathrm{H}$ dimerization of anionic oxacarbocyanines D-G (Scheme 1) in water is mainly driven by enthalpic contributions deriving from strong van der Waals attraction between the large and very polarizable chromophores in a cofacial structure [10]. Indeed, dyes F and G, bearing noncoplanar terminal phenyl groups which hinder the cofacial arrangement in the aggregate, have dimerization equilibrium constants lower by an order of magnitude than $\mathrm{D}$ and $\mathrm{E}$.

Relatively few cases of growth of cyanine J aggregates in ESAMs have been reported so far. These include only three negatively charged dyes: a benzimidocarbocyanine [11], a thiacyanine $[12,13]$ and the corresponding carbocyanine, 3,3' -disulfopropyl-5,5' -dichloro-9-ethyl-thiacarbocyanine, (THIATS) [12-15]. With respect to H-dimerization in water, the growth of J aggregates of cyanines in electrostatically self-assembled multilayers (ESAMs) with polycations is plausibly governed by a more complex combination of interactions involving the cyanine, the polycation in its different configurations, the small counterions, and the solvent. In a recent contribution concerning the formation of $\mathrm{J}$ aggregates in ESAMs, we have shown that the J-aggregation of dye $\mathrm{E}$ is very efficient on adsorbed polycations, but not upon interaction with the same polymers in solution [16]. Through the present contribution, we wish to further explore the aggregation behaviour of these cyanines upon interaction with three different polycations, both in solution and in ESAMs. Our ultimate goal is to establish whether a correlation can be traced for dyes D-G between the rather well-understood process of $\mathrm{H}$-aggregate formation in solution and the growth of J aggregates in ESAMs with polycations. The existence of such a correlation might help simplify the complex picture of the possible interactions leading to Jaggregate formation and single out the main features of the overall process.

\section{RESULTS}

\subsection{Dye aggregation in polycation solutions}

The effects of the interaction of the dyes D, F, and G with polycations poly(diallyl-dimethylammonium chloride) 


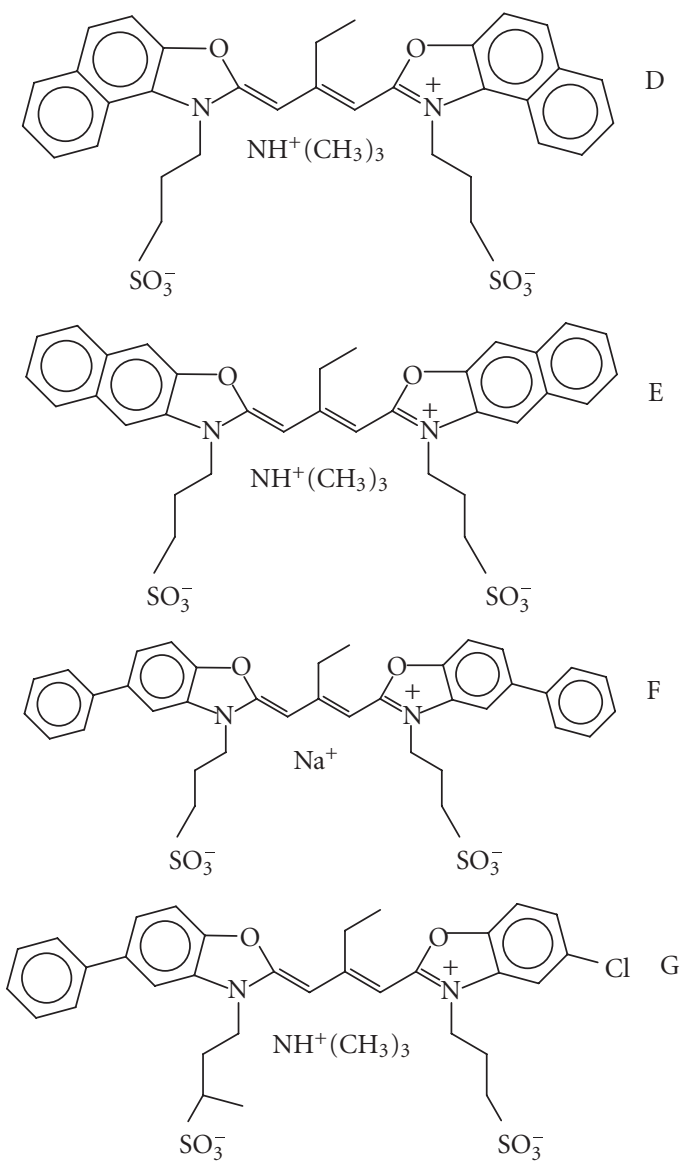

Scheme 1

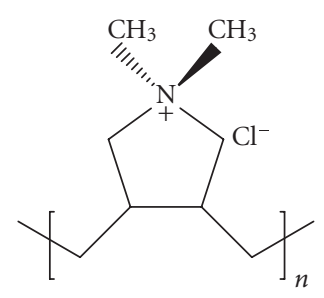

PDDA<smiles>CC(C)(C)CC(C[NH3+])C(C)(C)Cl</smiles>

PAH<smiles>CNCCC(C)(C)C</smiles>

PEI
Scheme 2

(PDDA, Scheme 2), poly(allylamine hydrochloride) (PAH) and polyethylenimine (PEI, protonated by addition of $\mathrm{HCl}$ ) on the UV-visible spectra are shown in Figure 1. The spectra in water (without added polycations, solid lines) feature absorptions due to the monomers $(\mathrm{M})$ and the H-dimers $\left(\mathrm{H}_{\mathrm{D}}\right)$ [10]; very weak shoulders on the absorption red edge are attributable to J aggregates.

Addition of excess polycation causes major changes in the absorption spectra thus demonstrating the occurrence of extensive dye/polyion interaction. Two types of spectra can be recognized. The first one, found for $\mathrm{D}$ and $\mathrm{G}$ with $\mathrm{PAH}$ and for $\mathrm{F}$ with all polycations, resembles the spectra in water, with a main band slightly red-shifted with respect to $H_{D}$ $(+4 \div 9 \mathrm{~nm})$ and a broad shoulder shifted by $+9 \div 13 \mathrm{~nm}$
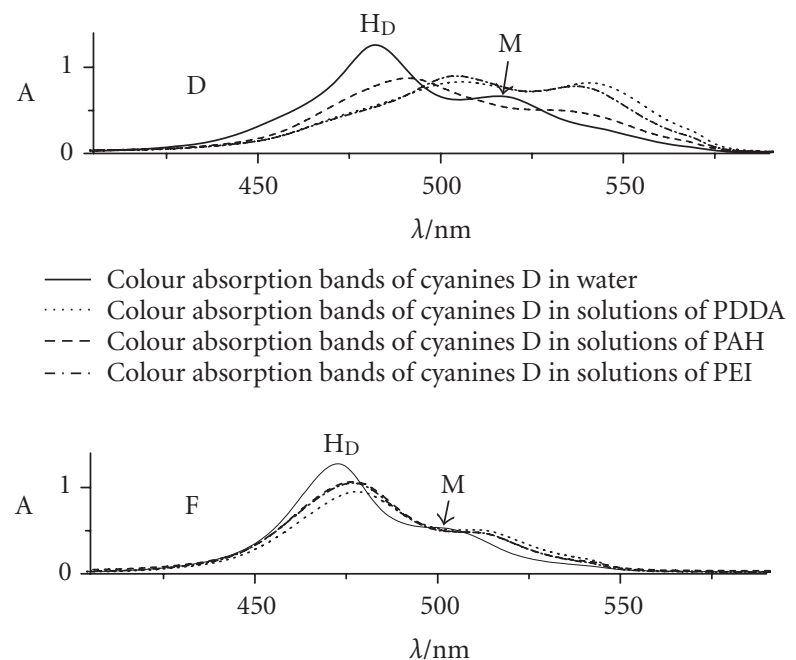

- Colour absorption bands of cyanines $\mathrm{F}$ in water

..... Colour absorption bands of cyanines F in solutions of PDDA

- - - Colour absorption bands of cyanines $\mathrm{F}$ in solutions of PAH

-. - Colour absorption bands of cyanines $\mathrm{F}$ in solutions of PEI

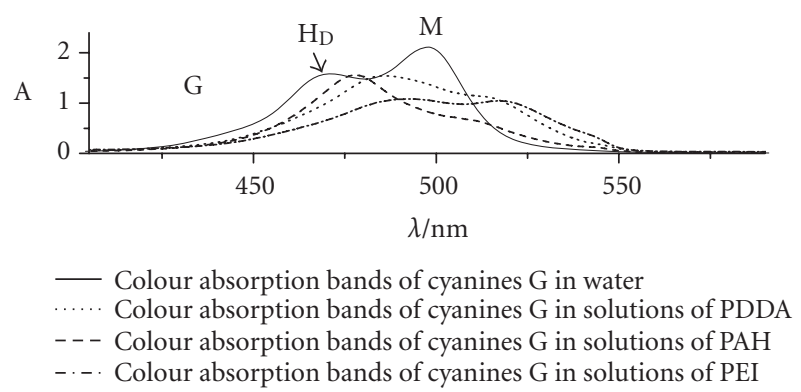

Figure 1: Colour absorption bands of cyanines D, F, and G in water (solid) and in solutions of PDDA (dotted), PAH (dashed), and PEI (dash-dotted). $\mathrm{H}_{\mathrm{D}}=\mathrm{H}$ dimers, $\mathrm{M}=$ monomers. Concentrations: dyes: $1.1 \times 10^{-5} \mathrm{M}\left(\mathrm{M}=\right.$ moles of monomer $\mathrm{dm}^{-3}$, throughout the paper) (D), $1.5 \times 10^{-5} \mathrm{M}$ (F and G); polycations: $2.5 \times 10^{-2} \mathrm{M}$. Optical path: $1 \mathrm{~cm}$. Spectra measured within $30 \mathrm{~min}-$ utes from dye/polycation mixing.

relative to the $\mathrm{M}$ band. This spectrum also closely resembles the spectrum of the H-dimer in water [10]. The second type of absorption, observed for D in PDDA and for D and G in PEI solutions, consists of two broad bands of similar heights more red-shifted than in the first type. This kind of spectrum was also found for cyanine E with PDDA [16].

In an attempt to gain insight into this complex aggregation pattern, we have performed a fluorescence steadystate analysis of two representative cases: D with PAH (onemaximum-one-shoulder spectrum) and D with PDDA (double-maximum spectrum). Emission and excitation fluorescence spectra of D/PAH and D/PDDA solutions are shown in Figure 2.

For D/PAH solutions the excitation spectra match fairly well the absorption one. The ratios of the maxima to the shoulders in both excitation and emission spectra show a little dependence (not larger than ca.10\%) on the monitoring and excitation wavelengths, respectively. Therefore, 

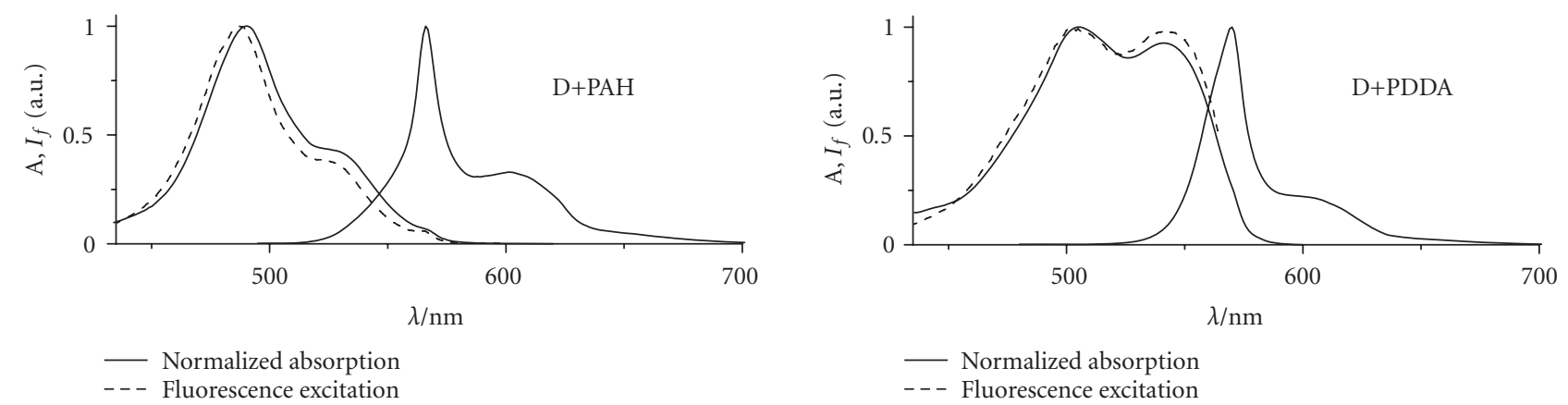

FIGURE 2: Normalized absorption (solid), fluorescence excitation (dashed), and emission spectra of cyanine D in PAH and PDDA solutions (same concentrations as for Figure 1$) ; \lambda_{\text {exc }}=490 \mathrm{~nm}(\mathrm{PAH})$ and $470 \mathrm{~nm}(\mathrm{PDDA}) ; \lambda_{\mathrm{em}}=610 \mathrm{~nm}(\mathrm{PAH})$ and $570 \mathrm{~nm}(\mathrm{PDDA})$.

absorption and fluorescence excitation spectra are attributable to a single species: we assign it to a small $\mathrm{H}$ aggregate whose absorption lies slightly to the red of the $\mathrm{H}_{\mathrm{D}}$ band in water, because of a change either in the polarity of its environment or, more probably, in the aggregate geometry. The emission spectrum combines a broad contribution from this species (maximum around $600 \mathrm{~nm}$ with a shoulder in the 550-570 nm region) [17] with the narrow J-aggregate emission (maximum at $566 \mathrm{~nm}, \mathrm{FWHM} \approx 300 \mathrm{~cm}^{-1}$ ). The latter is easily observed even when exciting at wavelengths within the other absorption bands: excitation energy transfer is efficient.

In the case of D/PDDA solutions, the emission and excitation spectra are independent of the excitation and monitoring wavelengths. Also, the excitation spectrum closely resembles the absorption one. So, we conclude that a single fluorophore is responsible for most of the absorption. It cannot be identified with either the monomer or the $\mathrm{H}$ dimer that dominate in water. In fact, these species have been observed in the presence of PDDA in much more diluted solutions; as found for the small $\mathrm{H}$ aggregate of $\mathrm{D}$ in the presence of $\mathrm{PAH}$, their absorption spectra were only slightly red-shifted (ca. +7 and $+4 \mathrm{~nm}$ for the dimer and the monomer) with respect to those in water. The fluorophore in Figure 2 is an aggregate since it is disrupted to form the monomer upon addition of ethanol. Its absorption/excitation spectra are unusual because of the two bands with similar intensities, one to the blue and the other to the red of the monomer band. These spectroscopic features are somewhere halfway between those of a pure $\mathrm{H} n$-mer and a pure $\mathrm{J} n$-mer and are predicted for an aggregate with long molecular axes, which roughly coincide with the local transition dipole moments, oblique to each other [18]. Again, in addition to the broad emission spectrum from this species, the narrow $\mathrm{J}$ band (maximum at $569 \mathrm{~nm}, \mathrm{FWHM} \approx 450 \mathrm{~cm}^{-1}$ ) is observed upon exciting at wavelengths within other absorption bands: J aggregates act as fluorescent exciton traps.

Apart from the difficult task of identifying the species that take part in aggregation equilibria which are established in aqueous solution with polycations, the present results confirm, with the dyes D, F, and G, what we found with $\mathrm{E}$ : interaction with polycations in water solution in no case favours formation of "standard" J aggregates. This is in contrast with what observed with pseudoisocyanine in solutions of poly(vinylsulfonate) [19] and with the thiacarbocyanine THIATS which is structurally analogous to dyes D-G; for the latter, a prominent $J$ band was obtained in the absorption spectrum upon interaction with PDDA and poly(phenylenvinylene) precursor, but not with PAH [15].

\subsection{J-aggregation in ESAMs}

As observed with cyanine E [16], compounds D, F, and G are adsorbed alternately with PDDA on a quartz support, yielding multilayers. Both dye and polycation adsorptions result in overcompensation of the film surface charge, thus enabling attachment of the oppositely charged species in the next step [20-23]. The absorption spectra of films made of 5 dye/PDDA bilayers per side of the plate are shown in Figure 3.

In all cases, the spectra of the wet films (solid lines) feature prominent $J$ bands (maxima at 570, 542, and $545 \mathrm{~nm}$ for $\mathrm{D}, \mathrm{F}$, and $\mathrm{G}$ ); some residual contribution from monomers, regular $\mathrm{H}$-dimers and, especially in the case of $\mathrm{F}$, distorted small aggregates is still observable in the shoulders towards shorter wavelengths. The steady-state fluorescence analysis, shown in Figure 4 for a D/PDDA wet bilayer, confirms J aggregates to be the dominant species in the film.

Drying of the multilayers causes an intensity decrease of all bands and the J band to broaden to some extent to the blue. The absorption intensity increases regularly with the number of adsorption cycles while the bleaching due to film drying becomes lower and lower: the film stability increases with its thickness. The spectral broadening indicates that the process of water removal causes an increase of disorder in the J aggregates grown on the film, with an associated decrease in the average exciton coherence length [24-26]. Dried films remain stable for weeks if kept in the dark; on the contrary, they undergo bleaching upon exposure to visible light. A marked hyperchromism of all ESAMs was observed upon increasing the ionic strength of the PDDA solution by addition of $\mathrm{NaCl} 0.5 \mathrm{M}$. Typical absorbance increases were between 30 and 100\%. Evidently, the added ions preferentially screen interchain repulsion in the polyelectrolyte leading to 

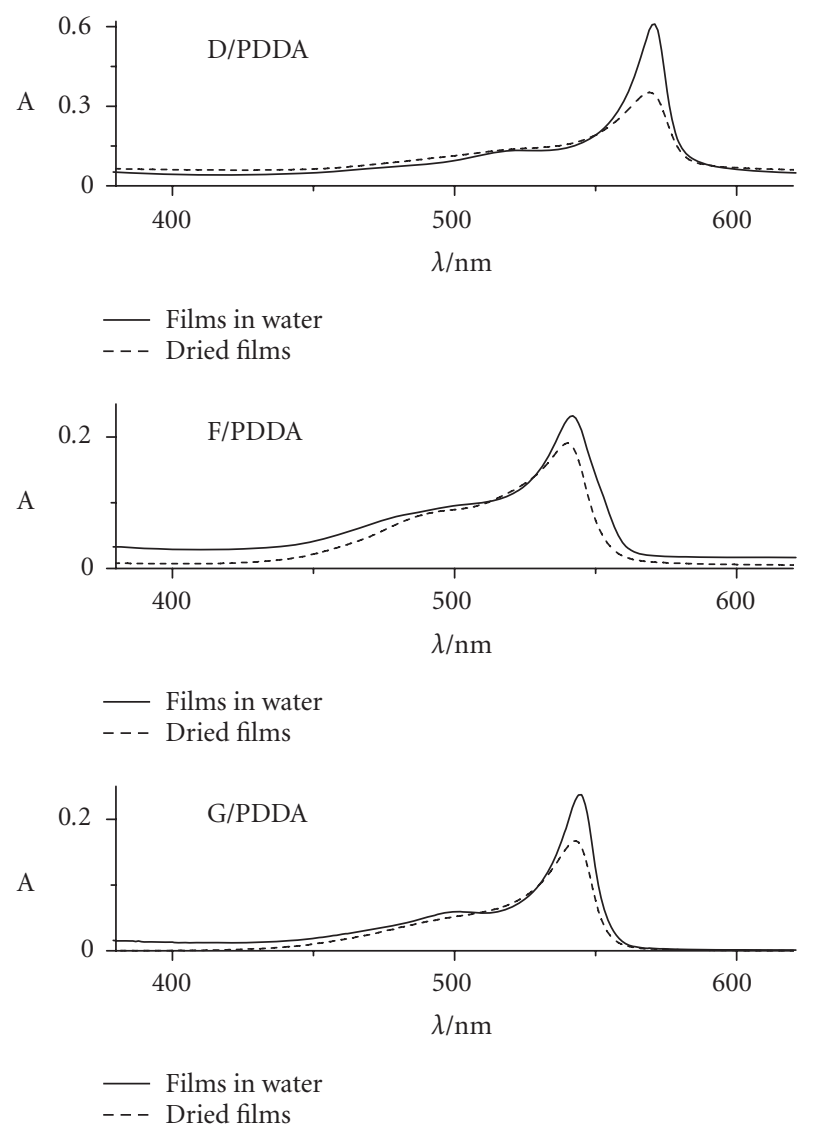

Figure 3: Absorption spectra of ESAMs (5 bilayers) of cyanines D, F, and $\mathrm{G}$ with PDDA. Films in water (solid lines); dried films (dashed lines).

a larger amount of adsorbed polyions [16]. An estimate of film thicknesses was obtained by Atomic Force Microscopy (AFM). Values for several (dye/PDDA $)_{5}$ multilayers obtained without added $\mathrm{NaCl}$ were rather scattered and ranged between 50 and $120 \AA$, with no clear and consistent differences between films with different dyes. A value around $100 \AA$ is expected for (E/PDDA $)_{5}$ [16]. Based on this "typical" thickness estimate, dye concentrations in the (dye/PDDA $)_{5}$ films were evaluated as described in the experimental section: we found similar values, ranging from 0.8 (dye G) to $1.1(\mathrm{~F}), 1.2$ (D), and $1.9 \mathrm{M}$ (dye E). Intriguingly these concentrations are of the same order of magnitude as those found in ESAMs of PDDA with a very different J-aggregating dye, tetrakis(4sulfonatophenyl)porphyrin diacid [27].

Information on the arrangement of the J aggregates within the dry ESAMs was provided by linear dichroism measurements (as a representative result, the case of a G/PDDA multilayer is shown in Figure 5). From the dichroic ratios $D=A_{\text {vert }} / A_{\text {horiz }}$ (the ratio of absorbances measured with light polarized vertically and horizontally) we have obtained the ensemble average $\left\langle\cos ^{2} \vartheta\right\rangle$ as

$$
\left\langle\cos ^{2} \vartheta\right\rangle=\frac{\cos ^{2} \beta D_{\beta}-D_{0}}{D_{\beta}\left(1-2 \sin ^{2} \beta\right)-D_{0}\left(1+D_{\beta} \sin ^{2} \beta\right)},
$$

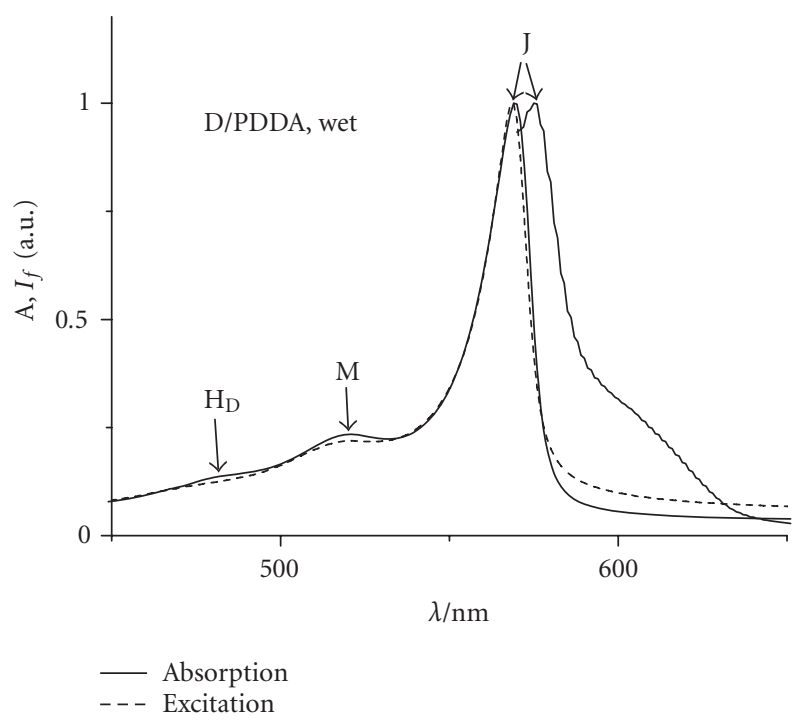

Figure 4: Absorption (solid), excitation (dashed), and emission spectra of a wet D/PDDA ESAM; 1 bilayer per side of the quartz plate; $\lambda_{\mathrm{exc}}=565 \mathrm{~nm}, \lambda_{\mathrm{em}}=580 \mathrm{~nm}$.

$\vartheta$ being the angle between the band dipole transition moment and the normal to the film plane and $\beta$ the incidence angle ( $\beta=0$, normal incidence) [16]. For the 5-bilayer films of $\mathrm{D}, \mathrm{F}$, and $\mathrm{G}$ with PDDA, we have measured $\mathrm{D}_{0}=1$ : the orientation of the J-aggregate dipole moments is totally isotropic in the film plane. Instead, we found $\mathrm{D}_{30^{\circ}}=1.2,1.3$, and 1.4 for D, F, and G, corresponding to values of $\left\langle\cos ^{2} \vartheta\right\rangle$ close to 0 . Thus, as already found for E, cyanines D, F, and $\mathrm{G}$ in their J aggregates tend to arrange with their transition dipole moments, roughly corresponding to their long molecular axes, lying in the film plane.

Even though all three cyanines yield large amounts of J aggregates in ESAMs, some differences are observed among them. The first one concerns the amount of dye adsorbed in the form of J aggregates in wet films: as shown in Figure 3, this is more than two times larger for D than for F, and G, which are adsorbed in comparable amounts. Corresponding data for dye E [16] indicate this to be adsorbed in amounts similar to D. A second difference is more subtle. After drying, a single D/PDDA bilayer gives absorption and fluorescence spectra similar to those shown in Figure 4 for the wet film, that is, with a very large contribution from J aggregates (although the relative $\mathrm{M}$ and $\mathrm{H}_{\mathrm{D}}$ intensities do increase). On the contrary, F/PDDA and G/PDDA single bilayers have very different absorption and fluorescence spectra whether wet or dry. When soaked with water, their spectral behaviour is similar to that of the wet D/PDDA single bilayer (Figure 4). But, after drying, their spectra change drastically (the case of F/PDDA is shown in Figure 6, that of G/PDDA is qualitatively alike). J aggregates are disrupted and monomers (with maxima shifted by ca. $+10 \div 15 \mathrm{~nm}$ with respect to water solutions, Figure 1) dominate the absorption and, even more, fluorescence spectra. It must be emphasized that such a J-aggregate disruption is almost limited to single 


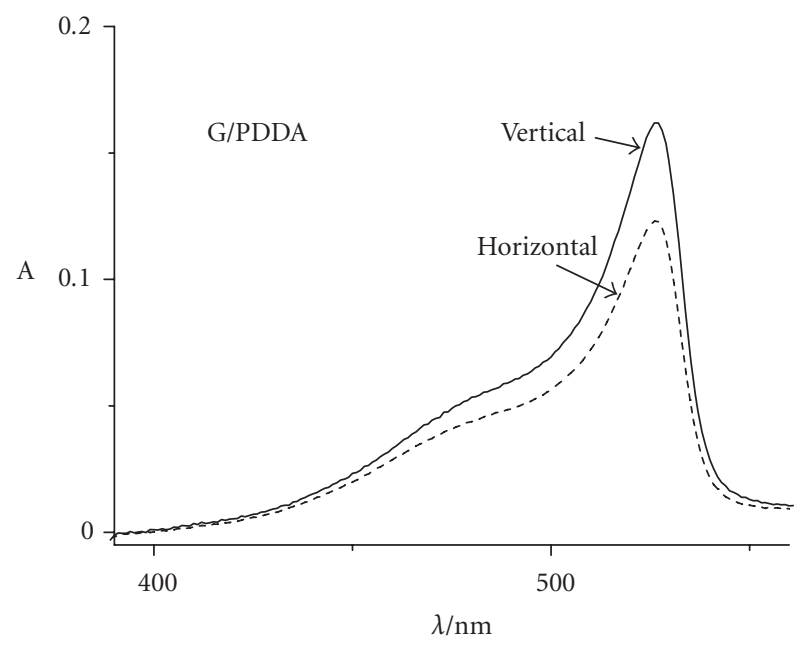

FIgURE 5: Absorption of a G/PDDA multilayer (5 bilayers per side of the quartz plate) measured with polarized light. Incidence angle $=30^{\circ}$ ( $\beta_{\text {app }}$, see the experimental section $)$.

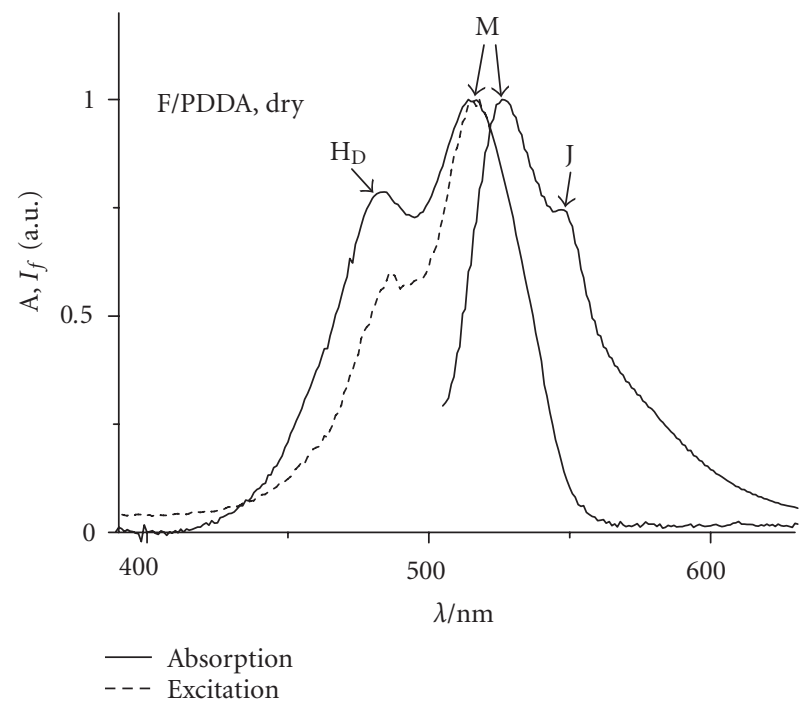

Figure 6: Absorption (solid), excitation (dashed, $\lambda_{\mathrm{em}}=530 \mathrm{~nm}$ ) and emission $\left(\lambda_{\mathrm{exc}}=500 \mathrm{~nm}\right)$ spectra of a dry F/PDDA ESAM; 1 bilayer per side of the quartz plate.

F and G/PDDA bilayers. As said above while commenting Figure 3, bleaching of J bands upon film drying becomes less and less pronounced with increasing number of deposition cycles.

A side observation of some interest is that the $\mathrm{H}$-dimer, while present in the dried film, contributes very little to fluorescence. This contrasts with the behaviour in water solution, where dimers had fluorescence quantum yields comparable with those of the monomers [17]. On the other hand, in that case such a similarity resulted from a drastic decrease in the rate of photo-induced twisting about one of the polymethine-chain bonds for the dimer. Likely, in ESAMs this deactivation path of the lowest excited singlet state is inhibited, because of steric hindrance by the environment, for both species; hence, the much larger radiative rate constant of $M$ relative to $H_{D}$ now results in a higher emission quantum yield.

\section{CONCLUDING REMARKS}

We have shown that interaction of four anionic carbocyanines with three polycations in aqueous solution and in ESAMs results in a complex aggregation pattern. In polycation solutions, at least two different kinds of aggregates have been observed in addition to minor amounts of the monomer and $\mathrm{H}_{\mathrm{D}}$ dimer. These unusual species are likely small aggregates with different geometries, plausibly distorted from the perfectly aligned pure $\mathrm{H}$ and J aggregates. No significant enhancement of standard J aggregation is promoted by interaction of the four cyanines with the three polycations in solution.

On the contrary, "classical" J aggregates of dyes D-G are extensively and almost exclusively formed on adsorbed PDDA. So, electrostatically adsorbed polycations provide excellent templates for selective J-aggregate growth of anionic oxacarbocyanines. In all cases, this process continues beyond compensation of the excess positive charge produced by polyion adsorption in the previous step, thus leading to an inversion of the surface charge. As a result, multilayers are easily obtained.

However, the amount of J aggregates incorporated, which is connected with the extent of surface charge overcompensation, is larger for cyanines D and E than for F and G. Also, single dye/PDDA bilayers of the two latter dyes, but not of the former ones, demonstrated their fragility by undergoing J-aggregate disruption upon water removal. If one combines these observations with the one order of magnitude lower dissociation constants of the $\mathrm{H}$ dimers of the first two dyes with respect to the latter in water [10], one is tempted to suggest the existence of a correlation between a stronger tendency to yield $\mathrm{H}$ aggregates in aqueous solution and that of forming more stable and abundant J aggregates in ESAMs. The differences in monomer/dimer equilibrium constants in water are mainly determined by larger enthalpic gains upon dimer formation in water for $D$ and $E$ relative to $F$ and $G$ [10]. These, in turn, are likely related with the structures of the four dyes, the first two having more extended and coplanar conjugated chromophores than the other two and being therefore able to assume more compact cofacial arrangements. According to such a view, attractive van der Waals interactions between the very polarizable dye molecules might still be of relevance in determining differences between the energetic aspects of J-aggregation in ESAMs. We may anticipate here, however, that it is the double anionic substitution on these dyes that is crucial for J-aggregate growth on charged surfaces. We have in fact observed other modes of aggregation, in water solutions as well as in ESAMs, for a number of well-known cationic cyanines which do not bear such a double substitution. The results concerning these dyes will be presented in a paper in preparation. 


\section{EXPERIMENTAL SECTION}

The oxacarbocyanines D, F, and G were obtained by courtesy of Ferrania Imaging Technologies (Italy) and were used as received (fluorescence analysis in solution revealed no emitting impurities in the experimental conditions adopted). Polymers poly (diallyl-dimethylammonium chloride) $\left(\mathrm{M}_{\mathrm{w}}=\right.$ 400000-500000) (PDDA), poly(allylamine hydrochloride) $\left(\mathrm{M}_{\mathrm{W}}=15000\right)(\mathrm{PAH})$, polyethylenimine $\left(\mathrm{M}_{\mathrm{W}}=750000\right.$, PEI) were purchased from Aldrich and were used without further purification. All solutions were prepared using deionized water (Millipore, Milli-Q, resistivity of $18.2 \mathrm{M} \Omega \cdot \mathrm{cm}$ ). PEI was made cationic by acidifying its solutions to $\mathrm{pH} \sim 3$ with $\mathrm{HCl}$.

Quartz SUPRASIL slides were purchased from Hellma. They were treated for 15 minutes in sulphochromic mixture (Carlo Erba Reagenti) and were sonicated for 1 hour in a solution containing $1 \% \mathrm{KOH}$ in a 1 : 20 water-isopropanol (Merck, p.a.) mixture [28], in order to remove all adsorbed organic impurities and make the quartz surface hydrophilic.

Electrostatically self-assembled films were prepared by dipping a slide alternately in solutions of the cationic polyelectrolytes (typically $3 \cdot 10^{-2} \mathrm{M}$ ) and, after rinsing, in the anionic dye solutions (ca. $10^{-4} \mathrm{M}$ ). Typical dipping times were 20 and 10 minutes, respectively. They had been shown to be long enough for the adsorption process to be near equilibrium with dye E [16]. After the last adsorption step, films were dried with warm air.

UV-Vis spectra were taken using a Varian Cary 100 Scan UV-Vis spectrophotometer. Linearly polarized light for dichroism measurements was obtained using dichroic sheet polarizers (Lot-Oriel). The incidence angle $(\beta)$ corrected for the refraction at the air-film surface was calculated as $\beta=\arcsin \left(n^{-1} \sin \beta_{\text {app }}\right)$, where $\beta_{\text {app }}$ is the apparent, macroscopic incidence angle and $n$ is the average refractive index of the film taken equal to 1.4 [19]. Excitation and emission fluorescence spectra were obtained with a JobinYvon-Spex Fluoromax-3 fluorometer.

The multilayer thicknesses were measured by AFM (CP Park Autoprobe, noncontact mode in air and at room temperature) after removing the films by peeling them off with a Scanning Tunnelling Microscope tip. Z-profiles across the scratches yielded the desired thickness values.

The dye concentrations in the films were determined by dissolving the multilayers in formamide and determining the dye amount spectrophotometrically $\left(\varepsilon_{\max }=\right.$ $145000,181000,162000$, and $188000 \mathrm{M}^{-1} \mathrm{~cm}^{-1}$ for $\mathrm{D}, \mathrm{E}, \mathrm{F}$, and $G$, resp.).

\section{ACKNOWLEDGMENTS}

This work was supported by the Grant FIRB RNBE01P4JF from the Italian Ministry of Education, University and Research (MIUR). The authors wish to thank Dr. Massimo Tonelli (CIGS, Modena) for his assistance with the AFM measurements.

\section{REFERENCES}

[1] E. E. Jelly, "Spectral absorption and fluorescence of dyes inthe molecular state," Nature, vol. 138, pp. 1009-1010, 1936.

[2] G. Scheibe, "Variability of the absorption spectra of some sensitizing dyes and its cause," Angewandte Chemie, vol. 49, p. 563, 1936.

[3] T. Kobayashi, Ed., J-Aggregates, World Scientific, Singapore, 1996.

[4] A. V. Vannikov, A. D. Grishina, B. I. Shapiro, et al., "Photoelectric, nonlinear optical and photorefractive properties of polyimide doped with J-aggregates of cyanine dye," Chemical Physics, vol. 287, no. 1-2, pp. 261-271, 2003.

[5] P. Schouwink, H. V. Berlepsch, L. Dähne, and R. F. Mahrt, "Observation of strong exciton-photon coupling in an organic microcavity," Chemical Physics Letters, vol. 344, no. 3-4, pp. 352-356, 2001.

[6] J. Wenus, L. G. Connolly, and D. G. Lidzey, "New organic materials and microcavity structures for strong exciton-photon coupling," Physica Status Solidi C, vol. 2, no. 11, pp. 38993902, 2005.

[7] Y. Sato, M. Furuki, M. Tian, I. Iwasa, L. S. Pu, and S. Tatsuura, "Improvement of on/off ratio in single-shot multichannel demultiplexing by using an optical Kerr gate of a squaryllum dye J aggregate film," Applied Physics Letters, vol. 80, no. 13, pp. 2254-2256, 2002.

[8] A. S. Kuch'yanov, R. V. Markov, A. I. Plekhanov, et al., "Passive mode locking of a $\mathrm{ND}^{3+}$ : YAG laser with a thin film of J-aggregates as a saturable absorber," Optics Communications, vol. 231, no. 1-6, pp. 343-348, 2004.

[9] G. Yağlioğlu, R. Dorsinville, and S. Özçelik, "Femtosecond response of J aggregates adsorbed onto silver colloid surfaces," Journal of Applied Physics, vol. 94, no. 5, pp. 3143-3146, 2003.

[10] I. Baraldi, M. Caselli, F. Momicchioli, G. Ponterini, and D. Vanossi, "Dimerization of green sensitizing cyanines in solution. A spectroscopic and theoretical study of the bonding nature," Chemical Physics, vol. 275, no. 1-3, pp. 149-165, 2002.

[11] S. Bourbon, M. Gao, and S. Kirstein, "Electroluminescence of self-assembled films of poly (p-phenylene vinylene) and J-aggregates," Synthetic Metals, vol. 101, no. 1, pp. 152-153, 1999.

[12] H. Fukumoto and Y. Yonezawa, "Layer-by-layer self-assembly of poly electrolyte and water soluble cyanine dye," Thin Solid Films, vol. 327-329, no. 1-2, pp. 748-751, 1998.

[13] N. Kometani, H. Nakajima, K. Asami, Y. Yonezawa, and O. Kajimoto, "Excited-state dynamics of the mixed J-aggregate of two kinds of cyanine dyes in layer-by-layer alternate assemblies," Chemical Physics Letters, vol. 294, no. 6, pp. 619-624, 1998.

[14] E. Rousseau, M. Van Der Auweraer, and F. C. De Schryver, "Steady-state and time-resolved spectroscopy of a self-assembled cyanine dye multilayer," Langmuir, vol. 16, no. 23, pp. 8865-8870, 2000.

[15] E. Rousseau, M. M. Koetse, M. Van der Auweraer, and F. C. De Schryver, "Comparison between J-aggregates in a selfassembled multilayer and polymer-bound J-aggregates in solution: a steady-state and time-resolved spectroscopic study," Photochemical and Photobiological Sciences, vol. 1, no. 6, pp. 395-406, 2002.

[16] A. Lodi and G. Ponterini, "J-aggregation of an anionic oxacarbocyanine in electrostatically self-assembled multilayers," Thin Solid Films, vol. 496, no. 2, pp. 585-594, 2006. 
[17] M. Caselli, L. Latterini, and G. Ponterini, "Consequences of Hdimerizatioin on the photophysics and photochemistry of oxacarbocyanines," Physical Chemistry Chemical Physics, vol. 6, no. 14, pp. 3857-3863, 2004.

[18] M. Kasha, H. R. Rawls, and M. Ashraf El-Bayoumi, "Molecular spectroscopy," in Proceedings of 8th European Congress on Molecular Spectroscopy, pp. 371-392, Butterworths, London, UK, 1965.

[19] M. L. Horng and E. L. Quitevis, "Excited-state dynamics of polymer-bound J-aggregates," Journal of Physical Chemistry, vol. 97, no. 47, pp. 12408-12415, 1993.

[20] N. G. Hoogeveen, M. A. Cohen Stuart, G. J. Fleer, and M. R. Böhmer, "Formation and stability of multilayers of polyelectrolytes," Langmuir, vol. 12, no. 15, pp. 3675-3681, 1996.

[21] J. B. Schlenoff, H. Ly, and M. Li, "Charge and mass balance in polyelectrolyte multilayers," Journal of the American Chemical Society, vol. 120, no. 30, pp. 7626-7634, 1998.

[22] K. Lowack and C. A. Helm, "Molecular mechanisms controlling the self-assembly process of polyelectrolyte multilayers," Macromolecules, vol. 31, no. 3, pp. 823-833, 1998.

[23] S. T. Dubas and J. B. Schlenoff, "Factors controlling the growth of polyelectrolyte multilayers," Macromolecules, vol. 32, no. 24, pp. 8153-8160, 1999.

[24] E. W. Knapp, "Lineshapes of molecular aggregates, exchange narrowing and intersite correlation," Chemical Physics, vol. 85, no. 1, pp. 73-82, 1984.

[25] H. Fidder, J. Knoester, and D. A. Wiersma, "Optical properties of disordered molecular aggregates: A numerical study," The Journal of Chemical Physics, vol. 95, no. 11, pp. 7880-7890, 1991.

[26] M. Van der Auweraer and I. Scheblykin, "One-dimensional J-aggregates: dependence of the properties of the exciton band on the model of the intermolecular coupling," Chemical Physics, vol. 275, no. 1-3, pp. 285-306, 2002.

[27] E. Collini, C. Ferrante, R. Bozio, A. Lodi, and G. Ponterini, "Large third-order nonlinear optical response of porphyrin Jaggregates oriented in self-assembled thin films," Journal of Materials Chemistry, vol. 16, no. 16, pp. 1573-1578, 2006.

[28] T. Schneider and O. D. Lavrentovich, "Self-assembled monolayers and multilayered stacks of lyotropic chromonic liquid crystalline dyes with in-plane orientational order," Langmuir, vol. 16, no. 12, pp. 5227-5230, 2000. 


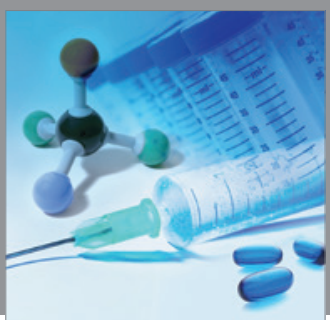

International Journal of

Medicinal Chemistry

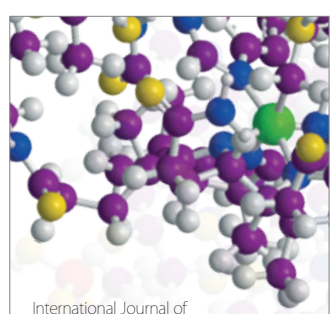

Carbohydrate Chemistry

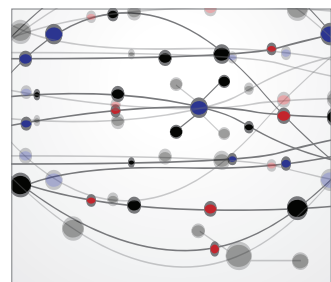

The Scientific World Journal
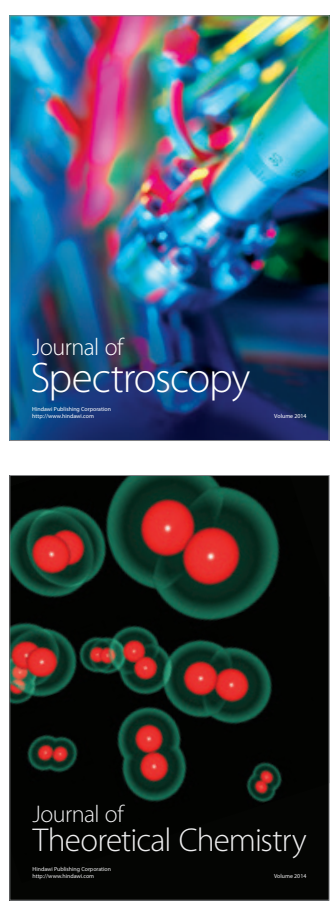
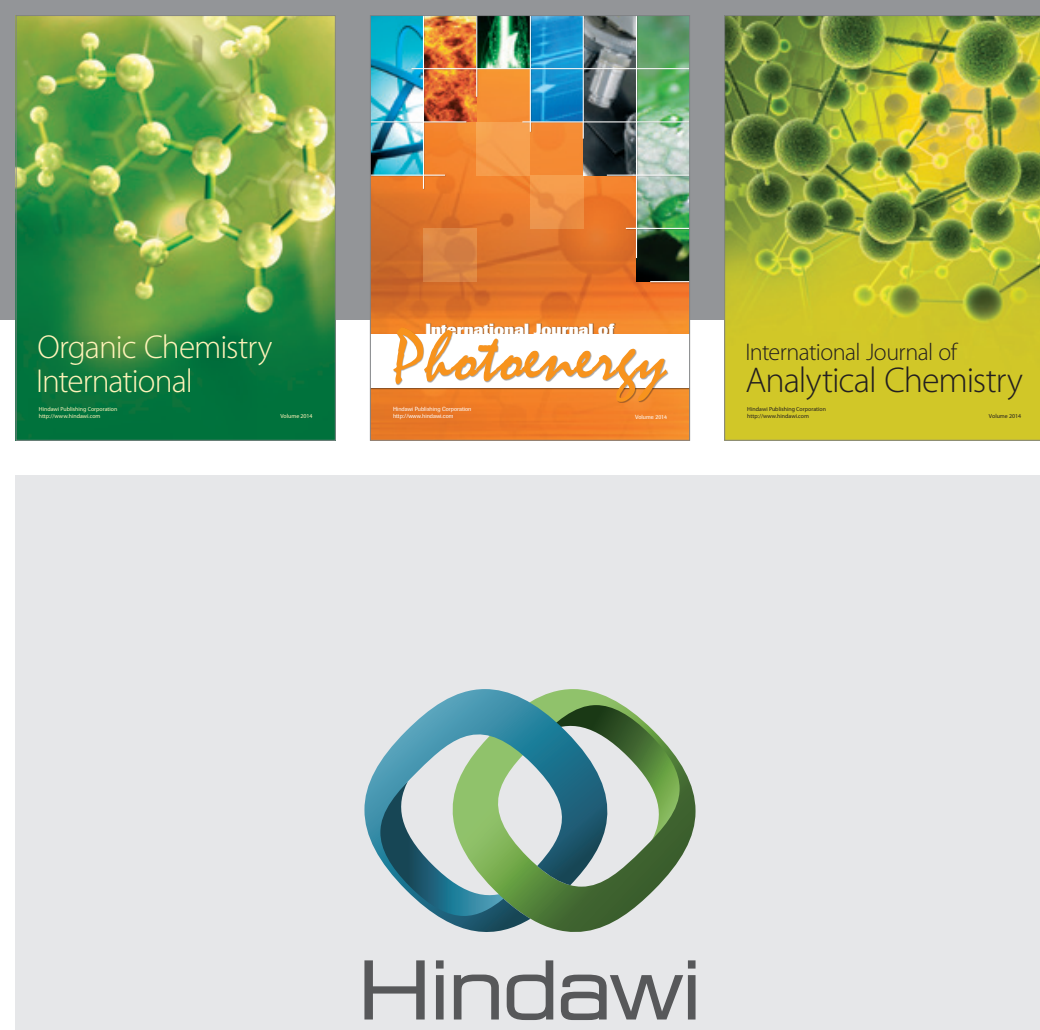

Submit your manuscripts at

http://www.hindawi.com
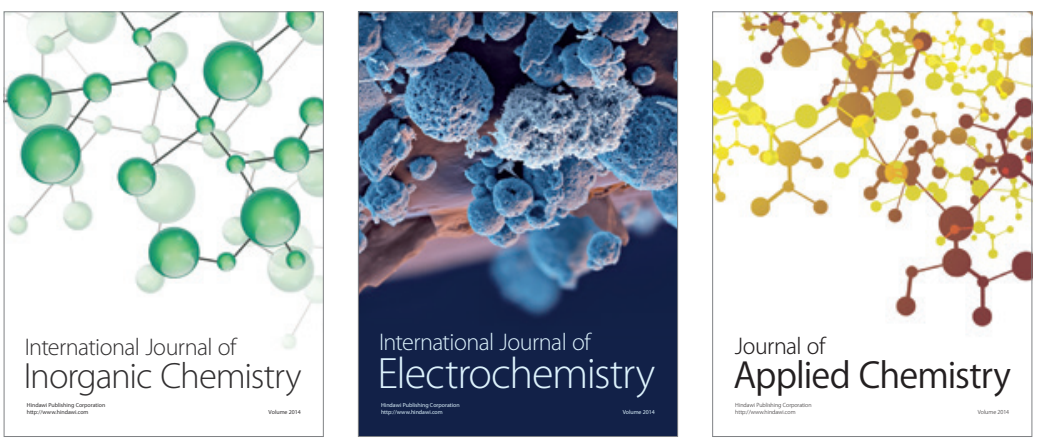

Journal of

Applied Chemistry
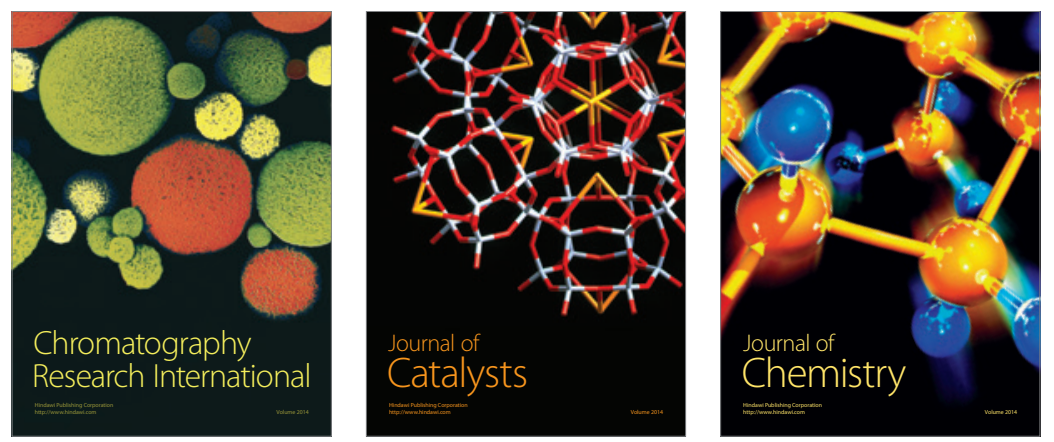
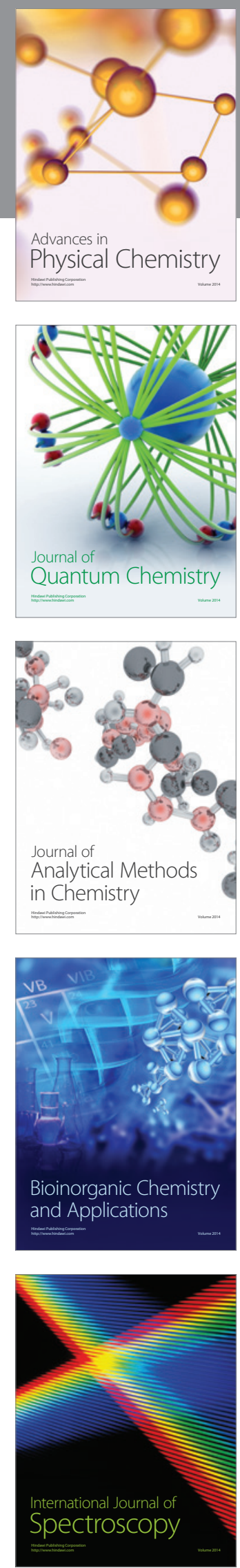